\title{
Carnets
}

Revue électronique d'études françaises de l'APEF

Deuxième série - 21 | 2021

Arts du vin

\section{Une poétique alchimique et cosmique : vigne, vin, alcool chez Gaston Bachelard}

Jean-Jacques Wunenburger

\section{(2) OpenEdition}

1 Journals

Édition électronique

URL : https://journals.openedition.org/carnets/12809

DOI : 10.4000/carnets. 12809

ISSN : 1646-7698

Éditeur

APEF

\section{Référence électronique}

Jean-Jacques Wunenburger, « Une poétique alchimique et cosmique : vigne, vin, alcool chez Gaston Bachelard », Carnets [En ligne], Deuxième série - 21 | 2021, mis en ligne le 31 mai 2021, consulté le 01 juin 2021. URL : http://journals.openedition.org/carnets/12809; DOl : https://doi.org/10.4000/ carnets. 12809

Ce document a été généré automatiquement le 1 juin 2021.

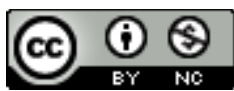

Carnets est mis à disposition selon les termes de la licence Creative Commons - Atribution - Pas d'utilisation commerciale 4.0 International. 


\title{
Une poétique alchimique et cosmique : vigne, vin, alcool chez Gaston Bachelard
}

\author{
Jean-Jacques Wunenburger
}

«Qu'est-ce que le vin? C'est un corps vivant où se tiennent en équilibre les "esprits" les plus divers, les esprits volants et les esprits pondérés, conjonction d'un ciel et d'un terroir $»^{1}$.

1 Gaston Bachelard (1884-1962) est un des rares philosophes à avoir cherché à approfondir en même temps les processus rationnels qui culminent dans l'esprit scientifique depuis le XVII siècle et se transforment de révolution en révolution, et les processus de l'imagination poétique, qui remontent aux plus anciens mythes. Du côté de la rationalité, il a mis en avant les obstacles épistémologiques suscités par l'image première, ses enracinements affectifs inconscients (Bachelard, 1938; 2000) et son historicité, passant d'une épistémè cartésienne à une autre newtonienne puis einsteinienne. Du côté de la poétique, il a mis en relief, par l'étude de l'alchimie et des poètes, la prégnance des éléments cosmologiques et la logique analogique et d'ambivalence dans la créativité de images de l'imaginaire.

2 Né en Champagne sèche (Bar-sur-Aube), ayant vécu et enseigné plus de 10 ans en Bourgogne (1930-1940), vivant au contact d'amis viticulteurs (Gaston Roupnel, devenu collègue de faculté, Gérard-Varet, futur recteur²), Bachelard ne pouvait délaisser la vigne ni le vin ni l'alcool en général dans sa panoplie des sources de rêveries (après avoir parlé aussi bien de la racine ou du lait.). On en trouve trace dans La formation de l'esprit scientifique (et dans La psychanalyse du feu, où la rêverie sur les substances apparait comme un frein à la formation de concepts chimiques, et surtout dans les textes ultérieurs sur la terre où le travail de la vigne et du vin vient clôturer les imaginaires du repos dans La terre et la rêverie du repos. On y retrouve toute la typologie d'un riche imaginaire avec ses invariants sémantiques et syntaxiques, sur fond d'une intuition herméneutique fondamentale : l'imaginaire de l'espace du dehors ouvre pour 
chaque sujet rêveur un "cosmos intime " ${ }^{3}$, lui-même riche de polarités contraires, enroulées autour d'un axe de verticalité à haute valeur symbolique ${ }^{4}$. L'apport irremplaçable des textes disséminés de Bachelard sur le vin vient de ce qu'il enrichit la «substance» du vin par ses multiples polarités, tout en l'insérant dans toute une trajectoire culturelle chargée de rêveries subjectives. Le vin est ainsi inséparable d'une chaine phénoménale qui commence par le plan de vigne et le cep à son commencement, qui finit dans la dégustation sensorielle d'un amateur en passant par toutes les étapes de la vinification, de la presse du raisin, sa maturation à la mise en bouteille. On peut donc, de manière synoptique, restituer plusieurs thématisations très éloquentes de l'imaginaire bachelardien du vin.

\section{1- Le travail vigneron}

3 Le vin n'est pas un produit spontané de la terre, mais aussi de la culture humaine. Le plant, sa culture, le raisin, sa cueillette puis son traitement, du pressoir à la cuve et au verre, est un travail, un métier qui suscite ses rites et ses mythes ${ }^{5}$. Bachelard, issu luimême d'une région rurale encore peu touchée par le progrès de la modernité industrielle, est familier de l'artisanat et du travail manuel de son époque ${ }^{6}$. Nourri depuis son enfance des souvenirs des travaux viticoles, Bachelard aime le vignoble comme transformation de la terre, ce mariage entre nature et culture, mais qui s'opère de manière respectueuse et sans aliénation du travailleur. C'est pourquoi lorsqu'il s'agira de décrire les imaginaires de la terre (après ceux du feu, de l'eau et de l'air), Bachelard renvoie l'imaginaire de la vigne et du vin à la rêverie du repos, pendant de la rêverie de la volonté active. Car si la vigne contraint au travail préalable, ce travail s'écarte de la polarité virile, agressive qui émane de l'ancrage du corps, du geste, de la main dans les métiers issus d'une volonté belliqueuse (forgeron, menuisier, etc). Le travail viticole se soumet aux promesses et aux rythmes du végétal et du sol, il est traversé par l' «anima» féminine plus que par l' «animus » masculin ${ }^{7}$; le vigneron prend soin, accompagne, allège, épure la force vitale de la nature, dans une posture que l'on nommerait de nos jours le « care ", le soin bienveillant, précautionneux, attentif, à la différence d'une imposition, maitrise, transformation violente et tranchée ${ }^{8}$. Le soin de la vigne deviendrait ainsi une image du prendre soin, appliquée à l'attention portée par l'homme, le vigneron, à la sauvegarde et l'épanouissement de tout autre vivant ${ }^{9}$.

\section{2- Le microcosme de la vigne et du vin}

4 Loin d'être un monde clos, refermé sur lui-même, la vigne (et le vin) se développent et se transforment au rythme du cosmos, et servent ainsi, pour Bachelard, de monde intermédiaire entre l'immensité infinie du ciel et l'obscurité cachée du sous-terrain. La vigne, dans son vignoble, concentre en elle les valeurs nutritives du sol, toujours singulières et les conditions climatiques favorables, toujours variables; dans la vigne se rejoignent, bien plus qu'ailleurs, la terre et le ciel. En ce sens Bachelard est proche de M. Heidegger, habitant dans le vignoble rhénan ${ }^{10}$, qui célèbre aussi dans son œuvre cette densité ontologique du sol, de ce qui y croît, la forêt, la clairière, en les subsumant sous des catégories cosmologiques et métaphysiques comme le "quadriparti ${ }^{11}$. La vigne et le vin sont un intermonde, relié vers le bas aux matières minérales du sous-sol mais aussi tourné vers le haut, les configurations célestes, astrales, changeantes avec le 
climat. La qualité singulière d'une récolte de raisin et du vin qui en est extrait, résulte bien toujours d'une conjonction rare et aléatoire entre les attributs de la terre et ceux de la météorologie et de la climatologie.

De sorte que le vin éveille dans l'imagination les attributs profonds de l'espace et du temps. De même que le vin reflète le sous-sol, et se définit par rapport à un lieu, de même il est tributaire des alignements et des événements cosmologiques. Bachelard signale en particulier l'importance d'une temporalité discontinue, fine, scandée de "kairos» (moments opportuns) et "rythmos", qui agissent toujours en des «lieux» uniques. C'est pourquoi le vigneron connait son vin par les particularités du terroir mais aussi par les calendriers astronomiques voire astrologiques qui discriminent les périodes favorables et défavorables.

Mieux que tout autre végétal, la vigne trouve l'accord des mercures de la terre donnant ainsi au vin son juste poids. Elle travaille tout le long de l'année en suivant la marche du soleil à travers tous les signes zodiacaux. Le vin n'oublie jamais, au plus profond des caves, de recommencer cette marche du soleil dans les «maisons " du ciel. C'est en marquant ainsi les saisons qu'il trouve le plus étonnant des arts : l'art de vieillir. D'une manière toute substantielle, la vigne prend à la lune, au soleil, à l'étoile un peu du soufre pur seul capable de bien « élémenter » tous les feux des vivants. Ainsi un vrai vin appelle le plus sensible des horoscopes.

S'il passe au ciel une comète, c'est une autre vendange! Nos formules, séchées dans les concepts, n'y voient guère qu'une étiquette pour illustrer la date d'un vin fameux, petite mnémotechnie d'un temps qui oublie la minutieuse individualité d'une année de loyal soleil. Mais le vigneron passionné, qui médite toute l'année les signes du vin, n'oubliera jamais que la comète nouvelle donne au vin une substance qui descend bien rarement du ciel sur la terre. La comète est moins un astre qu'une exhalaison. Cette longue queue molle coulant dans les hautes couches du ciel est essentiellement humide, elle est riche d'un feu liquide et suave, d'une eau essentielle et subtile, longuement distillée au firmament. La vigne attire cette eau céleste - la seule qu'elle tolère - venue des cieux dominateurs. Le vin de la comète en reçoit une douceur qui ne ruine pas la force (Bachelard, 1948 ; 1997 : 325-326).

Quant au lieu, il se prolonge dans le vin par l'intermédiaire des noms et des appellations géographiques, qui unissent les noms et les régions.

Mais la terre a ses gîtes pour les mots des diverses langues. Le vin ne saurait être un mot primitif dans les pays de la neige. Rien de plus local, rien de plus dialectal que le nom et que l'être des vins. Aux bords méridionaux où les raisins sont lourds, le vin rouge borde vraiment la mer méditerranéenne, grand empire du Milieu du règne dionysiaque. Ecrasés de culture classique, nous oublions le dionysisme de la vivacité, le dionysisme du vin blanc; nous ne rêvons pas devant des vins plus circonstanciés, les vins qui individualisent les coteaux (Bachelard, op.cit. : 331).

7 La culture vinicole viendrait donc illustrer les variations qualitatives intrinsèques $d u$ temps et de l'espace, de l'histoire du monde et de la géographie de la nature, que Bachelard oppose aussi bien à l'espace-temps géométrique abstrait qu'à l'espace-temps homogénéisant d'un de ses contemporains (H. Bergson), la durée continue, dont il ne cesse de se démarquer. La vigne et le vin apparaissent donc comme des rencontres rares de ces conjonctions cosmologiques, le bon lieu, au bon moment, qui nous éloignent des cadres du monde rationnel, économique, industriel de la modernité. 


\section{3- La vinification}

8 La préparation du vin, tout en obéissant aux lois de la chimie scientifique, se présente du point de vue de ses imaginaires oniriques comme analogue à l'alchimie et à ses métamorphoses (souffre, mercure, sel). L'alchimie que Bachelard avait étudiée (dans les archives de la bibliothèque municipale de Dijon) pour y saisir à l'œuvre les obstacles à la formation de la science moderne lui a révélé la richesse onirique des processus d'affinités des substances surdéterminées et des processus de transformation (solve et coagula). Sa symbolique et sa dynamique poétique deviennent pour Bachelard une matrice de notre expérience imaginative du vin et de l'alcool ; même plus la vinification se rapprocherait de l'archétype du processus alchimique, éclairant ainsi la puissance imaginaire du breuvage :

Jusque dans le détail de ses interminables recherches, l'Alchimie est toujours ambitieuse d'une grande vision du monde. Elle voit un univers en action dans la profondeur de la moindre substance ; elle mesure l'influence des forces multiples et lointaines dans la plus lente des expériences. Que cette profondeur soit finalement un vertige, que cette vue universelle paraisse une vision rêveuse quand on la compare aux principes généraux de la science moderne, voilà qui ne ruine pas la puissance psychologique de tant de rêveries convaincues, de si grandes images honorées d'une si constante conviction. Les belles matières: l'or et le mercure, le miel et le pain, l'huile et le vin, amassent des rêveries qui se coordonnent si naturellement qu'on peut y déceler des lois de rêve, des principes de la vie onirique. Une belle matière, un beau fruit nous enseignent souvent l'unité de rêve, la plus solide des unités poétiques. Pour un rêveur de la matière, un raisin bien composé n'est-il pas déjà un beau rêve de la vigne, n'a-t-il pas été formé par les forces oniriques du végétal ? Dans tous ses objets, la Nature rêve (Bachelard, op.cit. : 324).

Dans cette approche poétique, le vin résulte d'une rencontre d'opposés (coincidentia oppositorum) dont la conjonction harmonieuse crée une transmutation en une perfection, une sorte d'or vivant. Le rêve de l'alchimiste préfigure, miroite, dynamise aussi la rêverie devant le vin. Cette dimension alchimique de l'expérience éclaire alors toute une métaphorique partagée et transmise, notamment par les poètes. A titre d'exemple, le vin est d'abord l'opposé de l'eau. Non seulement le vin coupé devient une atteinte à sa nature mais dans le cycle végétal lui-même l'eau est déjà repoussée par la plante entière qui se protège dans ses racines contre tout mélange avec de l'eau. Par ailleurs, le vin se décline ensuite selon les deux métaphores du sang et de l'or.

Que de poètes, croyant ne vivre que dans un monde de métaphores, ont chanté le vin comme un sang végétal! L'alchimie parle d'un autre ton. C'est ici que la métaphore vraie montre toutes ses vertus de transaction. On dira aussi bien : le vin est le sang de la vigne ou le sang est le vin animal. Et entre les règnes extrêmes, entre les liquides extrêmes de haute noblesse, entre l'or potable et le sang, c'est le vin qui est le naturel intermédiaire. " Une quintessence, dit un vieux livre ${ }^{12}$, se joint volontiers à une autre quintessence. Il faut un véhicule ou médium à l'or qui est cette quintessence métallique pour se joindre à l'eau-de-vie végétale, et, par cette dernière, à l'homme ; car s'il y a une grande distance entre l'or et le vin, elle est encore plus grande entre l'or et l'homme, mais elle est petite entre le vin et l'homme, puisqu'il fait partie de sa subsistance. Il faut donc que l'or soit rapproché de la nature animale par les voies philosophiques et par l'esprit de vin qui le rendent universel... car autrement quelle apparence y aurait-il qu'un corps le plus compact (l'or)... puisse servir au rétablissement et à la conservation de la plus faible des créatures (Bachelard, op.cit. : 328). 
10 Ainsi l'imaginaire de la vigne et du vin échappe à toute inventivité subjective, à toutes associations conventionnelles. Bachelard a restauré la profondeur sémantique des poétiques du vin pour en déployer des lois oniriques aussi contraignantes que des lois physiques. Beaucoup d'études littéraires de l'imaginaire viticole trouveraient ainsi en Bachelard un réseau de motivation des images, ancré dans une expérience anthropologique et cosmologique.

\section{4- L'ivresse}

11 Enfin l'effet du vin nous transporte souvent dans des états de conscience modifiée, d'altération, de transformation biopsychique, qui ont pu justifier la méfiance, la défiance à l'égard du vin et souvent sa condamnation, sa prohibition (Islam). Mais ces états d'excès sont à leur tour soumis aux effets ambivalents : une ivresse d'élévation, d'intensification mentale et psychique et un autre inverse de désagrégation et de catatonie.

12 Cette dernière n'a guère invité Bachelard à des explorations de type psychopathologique. Il est notoire que Bachelard évoque peu les effets néfastes du vin, négligeant peut-être ainsi une ambivalence ultime, celles des expériences de consommation du vin. Il serait plutôt enclin à déplorer les campagnes anti alcooliques déjà de son époque ${ }^{13}$.

13 A l'inverse, Bachelard a particulièrement restitué la dimension stimulante de l'alcool en général qui ouvre des portes inconscientes qui vont conduire jusqu'à un élargissement de la rationalité, position surprenante chez un philosophe par ailleurs hautement rationaliste.

On se trompe quand on imagine que l'alcool vient simplement exciter des possibilités spirituelles. Il crée vraiment ces possibilités. Il s'incorpore pour ainsi dire à ce qui fait effort pour s'exprimer. De toute évidence, l'alcool est un facteur de langage. Il enrichit le vocabulaire et libère la syntaxe. En fait, pour en revenir au problème $\mathrm{du}$ feu, la psychiatrie a reconnu la fréquence des rêves du feu dans les délires alcooliques; elle a montré que les hallucinations lilliputiennes étaient sous la dépendance de l'excitation de l'alcool. Or la rêverie qui tend à la miniature tend à la profondeur et à la stabilité ; c'est la rêverie qui finalement prépare le mieux la pensée rationnelle. Bacchus est un dieu bon; en faisant divaguer la raison, il empêche l'ankylose de la logique et prépare l'invention rationnelle (Bachelard, 1938 ; 1949 : 328.).

14 Cette chaleur effervescente de l'alcool est liée en particulier à l'élément de la chaleur et $\mathrm{du}$ feu. L'alcool est feu et absorbe le feu pour en transsubstantier les propriétés calorifères.

Une des contradictions phénoménologiques les plus manifestes a été apportée par la découverte de l'alcool, triomphe de l'activité thaumaturge de la pensée humaine. L'eau-de-vie, c'est l'eau de feu. C'est une eau qui brûle la langue et qui s'enflamme à la moindre étincelle. Elle ne se borne pas à dissoudre et à détruire comme l'eau forte. Elle disparaît avec ce qu'elle brûle. Elle est la communion de la vie et du feu. L'alcool est aussi un aliment immédiat qui met tout de suite sa chaleur au creux de la poitrine : à côté de l'alcool, les viandes elles-mêmes sont tardives. L'alcool est donc l'objet d'une valorisation substantielle évidente. Lui aussi, il manifeste son action en petites quantités: il dépasse en concentration les consommés les plus exquis. Il suit la règle des désirs de possession réaliste : tenir une grande puissance sous un petit volume. 
Puisque l'eau-de-vie brûle devant les yeux extasiés, puisqu'elle réchauffe tout l'être au creux de l'estomac, elle fait la preuve de la convergence des expériences intimes et objectives. Cette double phénoménologie prépare des complexes qu'une psychanalyse de la connaissance objective devra dissoudre pour retrouver la liberté de l'expérience. Parmi ces complexes, il y a en un qui est bien spécial et bien fort ; c'est celui qui ferme pour ainsi dire le cercle : quand la flamme a couru sur l'alcool, quand le feu a apporté son témoignage et son signe, quand l'eau de feu primitive s'est clairement enrichie de flammes qui brillent et qui brûlent, on la boit. Seule de toutes les matières du monde, l'eau-de-vie est aussi près de la matière du feu (Bachelard, $1938 ; 1949$ : 146).

Et Bachelard de se remémorer les usages anciens du brûlot (le punch de Hoffman jusqu'au récent « vin chaud») et les nombreuses références aux poètes de Baudelaire à Novalis, en passant par Ed. Poe, Jean-Paul, Petitjean, qui l'incitent à inventer un « complexe de Hoffmann », configuration onirique du feu et de l'alcool chauffé.

Il serait sans doute souhaitable de compléter l'analyse bachelardienne en confrontant plus systématiquement les deux manières de vivre l'excès, dans ses deux versants en hyper et hypo, en transgression et régression (au sens des deux opposés à la «juste mesure » d'Aristote). Telle était la leçon des banquets grecs, soumis à la mania, la folie, le délire, mais que Platon déjà divisait en bonne et mauvaise ${ }^{14}$. On retrouverait ainsi l'ancienne sagesse biblique, qui voyait depuis Noé dans le vin une cause d'avilissement plus qu'une source d'augmentation des dons de l'âme. La divinité du vin loin de se réduire à une identité simple, est bien double, tel Janus, montrant deux visages en un UN. Comme nous l'avions résumé ailleurs :

Toute ivresse comporte une oscillation entre des extrêmes qui produit l'alternance entre une exubérance et un abattement, entre une phase d'explosion et une autre de dépression. L'ivresse est bien un vécu contrasté alternant joie et tristesse, au lieu de correspondre à une sorte de dilatation euphorique continue et homogène. ..On peut rechercher l'ivresse comme un état d'intensification de la vie par du vin, mais en étant conscient qu'elle nous fait accéder à une expérience plus turbulente et contradictoire qu'il n'y paraît (Wunenburger, $2008: 90$ ).

\section{Conclusion}

17 Ainsi pour Bachelard, la culture du vin, depuis le travail de la terre jusqu'à la dégustation du suc, ouvre sur un monde poétique complexe, véritable quintessence de l'imaginaire. La vigne est bien, comme le dit Heidegger, un quadriparti, une tétralogie, qui relie ensemble le bas et le haut, les humains et leurs rêveries de divinités (Dionysos, Bacchus); chaque expérience corporelle, du geste de planter ou tailler la vigne, jusqu'à l'art raffiné ou vulgaire de sa consommation, y gagne en intensité du fait des valeurs opposées qui s'y trouvent concentrées. Le vin devient bien une manière d'incorporer le cosmos en intériorisant et revivant le spectre des qualités, propriétés de la vie. Le vin, fruit de la terre et de son travail, ne se résume pas à ses manifestations factuelles, épuisées par la description ou l'explication scientifique. Il y a d'emblée un imaginaire du vin qui le met en relation avec de multiples dimensions de l'expérience du monde, et qui l'associe à une sorte d'élément mi-physique mi-psychique, que l'imagination active pour prendre possession de notre esprit, de nôtre âme et de notre corps. La divinité du vin devient le signe même qu'en lui s'exprime et s'expérimente une sorte de surréalité, de transcendance, qui nous arrache à soi et nous met en consonance avec le tout de la nature, visible et invisible. Le vin, avec ses strates de sensations, d'images, de symboles, 
peut donc être assimilé à un archétype, cette matrice signifiante et ambivalente d'une expérience du monde ${ }^{15}$.

\section{BIBLIOGRAPHIE}

BACHELARD, Gaston (1938; 2000). La formation de l'esprit scientifique. Paris : Vrin.

BACHELARD Gaston $(1938 ; 1949)$. La psychanalyse du feu. Paris : Gallimard, Idées.

BACHELARD, Gaston, (1948-1997). La terre et les rêveries du repos. Paris : Corti.

BACHELARD Gaston $(1960 ; 2016)$. La poétique de la rêverie. Paris : PUF.

MATTEI Jean-François (2001), Heidegger et Hölderlin, Le Quadriparti. Paris : PUF

PIERRON Jean-Philippe (2019). Prendre soin de la nature et des humains. Médecine, travail et écologie. Paris : Les Belles Lettres.

Platon (2012). Phèdre, 244a sq. Paris : Garnier-Flammarion.

WAVELET, Jean-Michel (2019). Gaston Bachelard, L'inattendu, les chemins d'une volonté. Paris :

L'Harmattan.

WUNENBURGER JEAN-JACQUES (2006). « Matérialisme tellurique et psychologie éristique de

l'imaginaire ", Bachelard et les éléments, Actes du colloque de Naples du 4 mai 2005, V.Chiore ed., in Bachelardiana, 2006-1, Genova: Il Melangolo ed., p. 141-150.

WUNENBURGER, Jean-Jacques (2008). « Variations sur les excès : de l'usage du vin socialisé » In Le vin et les rites, dir. J. Perard et M. Perrot, Oenoplurimedia : Chaire Unesco Dijon, pp. 85-90.

WUNENBURGER, Jean-Jacques (2012). Gaston Bachelard, poétique des images. Sesto S. Giovanni :

Mimesis.

WUNENBURGER, Jean-Jacques (2014). « Gaston Bachelard, Gilbert Durand, lecteurs de Jung », Cahiers Gaston Bachelard, n 13, E.U. Dijon.

WUNENBURGER, Jean-Jacques (2018). « L'imagination au travail : Bachelard, philosophe des sociétés pré-industrielles », « Imaginaire et praxis : autour de Baston Bachelard », Ethique, politique,

religion, revue de l'université Lyon3. Paris : Ed. Garnier, p. 169-185.

\section{NOTES}

1. Bachelard, La terre et les rêveries du repos, Corti, 1948, p 325. Citation reprise dans beaucoup de revues d'oenologie et de cuisine.

2. Sur ces relations voir la biographie de Jean-Michel Wavelet, Gaston Bachelard, L'inattendu, les chemins d'une volonté, L'Harmattan, 2019.p 70 sq. Gaston Roupnel, qui inspirera à Bachelard L'intuition de l'instant, (1932), Biblio-essais, 1994, était le fils d'un père modeste employé des chemins de fer Auguste Roupnel, chef de gare à Gevrey-Chambertin, normand d'origine et d'une mère bourguignonne, il vint vivre en Bourgogne dès l'âge de six ans. Élève au lycée de Dijon de 
1883 à 1891 puis à la Faculté de Dijon (histoire) de 1892 à 1895 puis enfin en Sorbonne, il ne fut pas reçu à l'agrégation malgré plusieurs tentatives. Il fut marié à une authentique vigneronne de Gevrey-Chambertin, Suzanne. Son fils Louis Roupnel se suicida à l'âge de 29 ans le 1/10/1937.

3. Voir Bachelard (1960; 2016).

4. Voir J.J. Wunenburger (2012).

5. Bibliographie d'une grande profusion.

6. Voir Wunenburger (2018).

7. Terminologie empruntée à C. G. Jung sur le psychisme féminisé et le psychisme virilisé. Voir notre analyse dans Wunenburger (2014).

8. Voir notre article Wunenburger (2016).

9. Voir Jean-Philippe Pierron (2019).

10. Les vins du Rhin ont inspiré une part du romantisme allemand.

11. Sur le quadriparti de Martin Heidegger voir Jean-François Mattéi (2001).

12. Le Crom, Vade-mecum philosophique... en faveur des Enfants de la Science, Paris, 1718, p. 88 (référence de Bachelard dans le texte).

13. Pour Bachelard (1949:153), «Le brûlot et le punch sont actuellement dévalorisés. L'antialcoolisme, avec sa critique tout en slogans, a interdit de telles expériences ».

14. Platon (2012: 244a sq).

15. Bachelard va même jusqu'à esquisser une psychologie des tempéraments basée sur les 4 éléments, et donc une psychologie de la terre, à laquelle appartient l'amateur des rêveries du vin. «Si notre présent travail pouvait avoir une utilité, il devrait suggérer une classification des thèmes objectifs qui préparerait une classification des tempéraments poétiques. Nous n'avons pas encore pu mettre au point une doctrine d'ensemble, mais il nous semble bien qu'il y a quelque rapport entre la doctrine des quatre éléments physiques et la doctrine des quatre tempéraments. En tout cas les âmes qui rêvent sous le signe du feu, sous le signe de l'eau, sous le signe de l'air, sous le signe de la terre se révèlent comme bien différentes. En particulier, l'eau et le feu restent ennemis jusque dans la rêverie et celui qui écoute le ruisseau ne peut guère comprendre celui qui entend chanter les flammes: ils ne parlent pas la même langue » (Bachelard, $1938 ; 1949$ : 154).

\section{RÉSUMÉS}

Le vin comme d'autres alcools et drogues distille un imaginaire culturel de la substance végétale, dont les polarités opposées semblent le signe d'un archétype. Né des propriétés naturelles et symboliques de la vigne, le vin est le fruit d'une habile fermentation qui allie travail de substances et travail du vigneron. De la production à la consommation, le vin est le fruit d'une dialectique subtile de contraires, eau et vin, terre et ciel, or et sang, chaud et frais, universel et singulier, largement déployée dans la rêverie, comme l'illustre Gaston Bachelard. On peut même élargir cette bipolarité jusque dans les rites sociaux où coexistent les contraires du dionysisme/ christianisme sacré et de l'ivresse noachite avilissante. Si le vin est inséparable dans la mythologie de Dionysos/Bacchus, ce dieu de l'ivresse est semblable alors à Janus, divinité aux deux visages.

As many other alcohols and drogues, wine distils a cultural imaginary of the vegetable substance, whose opposite polarities seem to be the sign of an archetype. Sprout from the natural and 
symbolic properties of the vineyard, wine is the fruit of an experienced fermentation process that puts together transformation of substances and the wine-grower's expertise. From production to consumption, wine is the fruit of a subtle dialectic of contraries such as water and wine, Earth and sky, gold and blood, hot and cool, universal and singular. All this dialectic has been largely developed in reverie by the eminent Gaston Bachelard.

We could even expand this polarity to social rites in which coexists the opposition between the Dionysus cult/ holy Christianism and the Noachite degrading inebriation.

If wine is inseparable from the image of Dionysus/Bacchus, this God of the inebriation is alike to Janus, as God of the duality.

INDEX

Keywords : vineyard, wine, transformation process, substance, inebriation

Mots-clés : vigne, vin, travail, substance, ivresse

\section{AUTEUR}

\section{JEAN-JACQUES WUNENBURGER}

Université Jean Moulin Lyon3

jean-jacques.wunenburger[at]wanadoo.fr 